\title{
Electrophysiological and behavioral evidence reveals the effects of trait anxiety on contingent attentional capture
}

\author{
Yi-Chun Tsai ${ }^{1}$ - Hsin-Jie Lu ${ }^{1}$ • Chi-Fu Chang ${ }^{1}$ - Wei-Kuang Liang ${ }^{1}$. \\ Neil G. Muggleton ${ }^{1,2,3}$ • Chi-Hung Juan ${ }^{1}$
}

Published online: 27 June 2017

(C) Psychonomic Society, Inc. 2017

\begin{abstract}
Few studies have investigated the effects of anxiety on contingent attentional capture. The present study examined contingent attentional capture in trait anxiety by applying a rapid serial visual presentation (RSVP) paradigm during electroencephalographic recording. Overall, the behavioral and electrophysiological results showed a larger capture effect when a distractor was the same color as the target compared to when the distractor was not of the target color. Moreover, high-anxiety individuals showed a larger N2pc in the target colored distractor condition and nontarget colored distractor condition compared to the distractor-absent condition. In addition, the reaction time was slower when distractors were presented in the left visual field compared to when they were in the right visual field. This pattern was not seen in lowanxiety individuals. The findings may indicate that highanxiety individuals allocate attention to the target less efficiently and have reduced suppression of distractors compared to low-anxiety individuals who could suppress attention to the distractors more efficiently. Future work could valuably investigate the consequences of such differences in terms of benefits and disruption associated with attentional capture differences in a range of anxious populations in different risk monitoring situations.
\end{abstract}

Yi-Chun Tsai and Hsin-Jie Lu contributed equally to this work.

Chi-Hung Juan

chijuan@cc.ncu.edu.tw

1 Institute of Cognitive Neuroscience, National Central University, No.300, Jhongda Rd, Jhongli 320, Taiwan

2 Institute of Cognitive Neuroscience, University College London, London, UK

3 Department of Psychology, Goldsmiths, University of London, London, UK
Keywords Contingent attentional capture · Trait anxiety · N2pc $\cdot$ Suppression

Attention is a critical capability of human beings to allocate and process relevant information in the world. It was first classified by James (1890) into a passive (involuntary) and active (voluntary) system. A century later, classification to understand the neural systems was proposed that differentiated the subject's goal (goal directed) and salient stimuli (stimuli driven) as the components that affect our attentional control system. In goal-directed or top-down processes, attention is allocated to selecting objects from the environment related to current goals while ignoring other goal-irrelevant objects. For instance, if we want to look for a friend wearing a red coat in a crowd, then we will focus on people with red clothes and filter out irrelevant colors. In stimulus-driven or bottom-up processes, a salient unexpected stimulus irrelevant to the intentions of the observer can capture attention. For example, when we walk on the street and suddenly hear a loud noise, our attention will be captured by this salient noise (e.g., Posner \& Petersen, 1990; Corbetta \& Shulman, 2002; Ruz \& Lupiáñez, 2002).

One way to study the interrelation between goal-directed and stimulus-driven processes is by manipulating the degree of attentional capture in visual tasks. Attentional capture occurs when a salient unexpected stimulus irrelevant to the intentions of the observer seizes attention and affects the efficacy of target detection. As an example, during performance of a visual-search task, an irrelevant item suddenly appearing on the opposite side of the screen to the target can result in an increased response time for target detection.

Attentional capture was previously thought to be predominantly affected by bottom-up processes in the brain. However, several studies have recently demonstrated that 
top-down processes can frequently modulate attentional capture (Corbetta, Patel \& Shulman, 2008; Corbetta \& Shulman, 2002; Serences et al., 2005; cf. Sawaki, Geng, \& Luck, 2012). For instance, one experiment showed that the subject's attention when searching for a red cross was modulated by the set of features relevant to searching for this target. In this case, this led to a red circle affecting performance (Bacon \& Egeth, 1994; Folk, Remington \& Johnston, 1992; Gibson \& Kelsey, 1998; Yantis \& Jonides, 1990). This type of effect demonstrates that our attention is attracted to stimuli sharing targetrelevant features and is termed contingent attentional capture and neuroimaging studies have led to the hypothesis that contingent attentional capture is modulated by the interaction of bottom-up processes and the top-down control system (Serences et al., 2005).

The relation between various psychiatric disorders, including anxiety, and the attentional system been frequently examined. For example, it has been shown that attentional capture is influenced among populations with a high rate of anxiety (Macleod \& Mathews, 1991; Williams, Watts, MacLeod, \& Mathews, 1988) and that high-anxiety individuals are less efficient in filtering task-irrelevant distractors during visualspatial working memory or visual search tasks, even when these distractors are emotionally neutral (Moser, Becker, \& Moran, 2012; Qi, Ding, \& Li, 2014). Nevertheless, most studies have examined the automatic attentional capture system while the relation between anxiety and contingent attentional capture still remains unclear.

More recently, there have been several hypotheses proposed on the relationship between anxiety and the attentional control system. For instance, Eysenck, Derakshan, antos, and Calvo (2007) proposed the attentional control theory (ACT) that states that anxiety impairs attentional control processing and efficiency. They hypothesized that anxiety disrupts the balance between the top-down and bottom-up attentional control systems by increasing the influence of the bottom-up attentional system and decreasing the influence of the top-down control attentional system (Derryberry \& Reed, 2002; Eysenck et al., 2007; Sylvester et al., 2012). This theory leads to the possibility that the contingent attentional capture system in anxious people is less active due to such a decrease in the top-down system that is involved in target selection. Consequently, this would mean that anxious populations cannot process targets efficiently during attention-demanding tasks because of an inefficient adoption of the set of features relevant to target search. Additionally, an increased number of distractors could capture their attention, so disrupting task performance, due to increased influence of the bottom-up system.

Furthermore, Sawaki and Luck (2010) proposed the signal suppression hypothesis of controlled attention capture, which states that when the task-irrelevant stimuli do not share similar characteristics with the target, a suppression mechanism is evoked, preventing attention being captured by the distractors. This hypothesis infers that task-irrelevant stimuli that do share similar characteristics are commonly permitted to capture our attention. Interestingly, two independent fMRI studies observed a correlation between high-trait-anxiety individuals and prefrontal cortex activity, a region known to be involved in the regulation of top-down processes, which in turn modulates the suppression mechanism (Rissman \& Zanto, 2015). In one of the studies, high-trait-anxiety individuals showed a decrease of activity in the prefrontal cortex and slower reaction times during a response-conflict task (Bishop, 2009). The other study found that the structural integrity and connectivity of the amygdala-prefrontal cortex pathway is inversely proportional to high levels of trait anxiety (Kim \& Whalen, 2009). The abovementioned studies suggest that a decreased effect of the suppression mechanism might allow attention to be captured by increased numbers of distractors. Altogether, the previous studies offer a framework and basis for examining contingent and automatic attentional capture and how it is influenced by the interplay between the top-down and bottomup systems.

Unlike anxiety and its relationship with contingent attentional capture, the study of the attentional capture control system and its relationship with the asymmetries of visual attention have been widely discussed in previous research. For instance, numerous studies have found a general leftward attentional bias for spatial attention under voluntary control (Corbetta \& Shulman, 2002; Du \& Abrams, 2010). Furthermore, Du and Abrams (2010) examined and spatially differentiated stimulus-driven attentional capture from contingent attentional capture with classic visual search and rapid serial visual presentation (RSVP) tasks, revealing that contingent attentional capture is an asymmetric process unlike stimulus-driven automatic attentional capture. While few studies have examined the effects of anxiety on the asymmetrical involuntary attention control processes, a set of studies based on the attentional control theory (Eysenck et al., 2007) showed that anxiety increases the activation of the rightly lateralized ventral network and decreases the activation of the dorsal frontal network (e.g., Chang et al., 2013; Corbetta $\&$ Shulman, 2002). These activity modulations resulted in a suppression reduction of relevant distractors suggesting that the right hemisphere dominance of the ventral network and its increased activation might lead to a leftward visual field asymmetry effect in the high-trait-anxiety group. It is worth noting that several studies examining attentional systems in human subjects commonly employ the rapid serial visual presentation (RSVP) task in conjunction with electroencephalography (EEG) to have a better understanding of attentional capture (Chang, Liang, Lai, Hung, \& Juan, 2016). Particularly, the $\mathrm{N} 2 \mathrm{pc}$ (N2 posterior contralateral) event related potential (ERP) component is often used to investigate processing related to spatial attention in visual search since it reflects the 
selection of the target and suppression of irrelevant and competing distractors (Eimer, 1996; Luck \& Hillyard, 1994; Woodman \& Luck, 1999).

Previous studies have shown that contingent attentional capture is an asymmetric process (Du \& Abrams 2010) and that anxiety increases activation of rightly lateralized networks (Vrticka, Sander, \& Vuilleumier, 2013); however, it is still presently unknown if anxiety affects the lateralized contingent attentional capture system.

The present study aimed to both investigate the effect of anxiety on the contingent attentional capture system and its relationship with involuntary asymmetrical attention. We employed a RSVP task along with EEG recordings to investigate the effects of individual differences in trait anxiety on contingent attentional capture. Furthermore, we measured the $\mathrm{N} 2 \mathrm{pc}$ component from the event-related potential (ERP) to identify the processing related to spatial attention in task performance and correlate it with individuals' trait anxiety. This was carried out to assess whether increased anxiety was associated with altered contingent capture due to affected topdown target-related processing (i.e. poorer ability to detect the target due to poorer modulation of behavior relevant to the target attributes) or a reduced ability to suppress automatic capture by distractors sharing attributes with the target.

We expected that in high trait-anxiety individuals, control processing and efficiency would be altered, resulting in reduced target processing efficiency and more distraction by target-relevant distractors. As a result, an increase of the contingent attentional capture effect was predicted. Moreover, we also expected that the N2pc might be greater in a high traitanxiety group relative to a low trait-anxiety group. Finally, visual asymmetry was expected to play a role in efficiency for allocating targets when a distractor was located in the left visual field.

\section{Materials and method}

\section{Participants}

A group of 454 students from National Central University performed the Chinese version of the State and Trait Anxiety Inventory-Trait (Chinese version of STAI-T; Chung \& Long, 1984) survey. Twelve participants were excluded due to omitting responses in the STAI-T questionnaire, leaving answers from a total of 442 participants for evaluation. From these, our study included participants with scores within the upper $20 \%$ (score $\geq 52,80$ th percentile) and the lower $20 \%$ (score $\leq 34,20$ th percentile) of the STAI-T questionnaire scores. Twenty-four participants were in the high-traitanxiety (HTA) group (12 males; mean age $=18.83$ years, range: 18-19), with STAI-T scores ranging from 52 to 72 $(M=58.83, S D=5.58)$, and with STAI-S score ranging from
20 to $78(M=55.83, S D=14.58)$. Twenty-four participants were in the low-trait-anxiety (LTA) group (12 males; mean age $=18.17$ years, range: $18-20)$, with STAI-T scores ranging from 23 to $34(M=28.96, S D=0.48)$, and with STAI-S score ranging from 20 to $48(M=29.96, S D=6.14)$. These 48 participants were all right-handed, with normal/corrected vision, and without any neurological diseases. All participants gave informed consent prior to participating and the study was approved by the local ethics committee.

\section{Apparatus and materials}

The experimental task (Fig. 1) was adopted from Chang et al. (2013; Chang et al., 2016). A RSVP of groups of three letters was presented against a white background on a 23-inch LCD monitor with a $120 \mathrm{~Hz}$ vertical refresh rate. Each trial consisted of 25 frames. Each frame contained three uppercase letters, such that there was one letter on the left, one in the center, and one on the right, and was presented for $50 \mathrm{~ms}$, followed by a $16.7 \mathrm{~ms}$ blank interval, such that a frame was presented every $66.7 \mathrm{~ms}$. Letters were randomly selected from the English alphabet and occurred in a sequence without replacement. Each letter was $1^{\circ} \times 1.3^{\circ}$ in size. In the central letter stream, a single red (Commission International del Eclairage [CIE]; $x=0.60, y=0.34)$ target letter was embedded amongst the other colored letters, which were green (CIE $x=$ $0.28, y=0.58)$, blue $(x=0.17, y=0.13)$, purple $(x=0.25, y=$ $0.14)$, and cyan $(x=0.20, y=0.15)$. Colors in the array were isoluminant $\left(22 \mathrm{~cd} / \mathrm{m}^{2}\right)$. Across trials, the target appeared randomly between the 15 th and 20th frames and was selected equally from the first eight and last eight letters of the alphabet ( $A$ to $H, S$ to $Z$ ). The peripheral distractor streams were located $3^{\circ}$ to either side of the central target stream. In one third of the trials, the letters of the peripheral distractor streams were gray in color (distractor absent). In another one third of the trials, four of the peripheral distractor letters were red (target-colored distractor; TC), and in the remaining one third of the trials four of the peripheral distractor letters were green (nontarget colored distractor, NTC). These four colored distractors were presented during the period from $133.4 \mathrm{~ms}$ before the target letter onset to $66.7 \mathrm{~ms}$ after the target letter offset.

The State-Trait Anxiety Inventory was used to assess participant's anxiety level. It is a self-report measurement scale that consists of two parts with each part comprised of 20 items. The first part is the state version of the State-Trait Anxiety Inventory (STAI-S), which requires participants to answer questions regarding their current feelings. The second part is the trait version of the State-Trait Anxiety Inventory (STAI$\mathrm{T})$, which requires participants to answer regarding their general feelings. A 4-point response scale is employed ( $1=\mathrm{sel}$ dom/never, 4 = very often/always), and the higher scores indicate a more severe anxiety. 


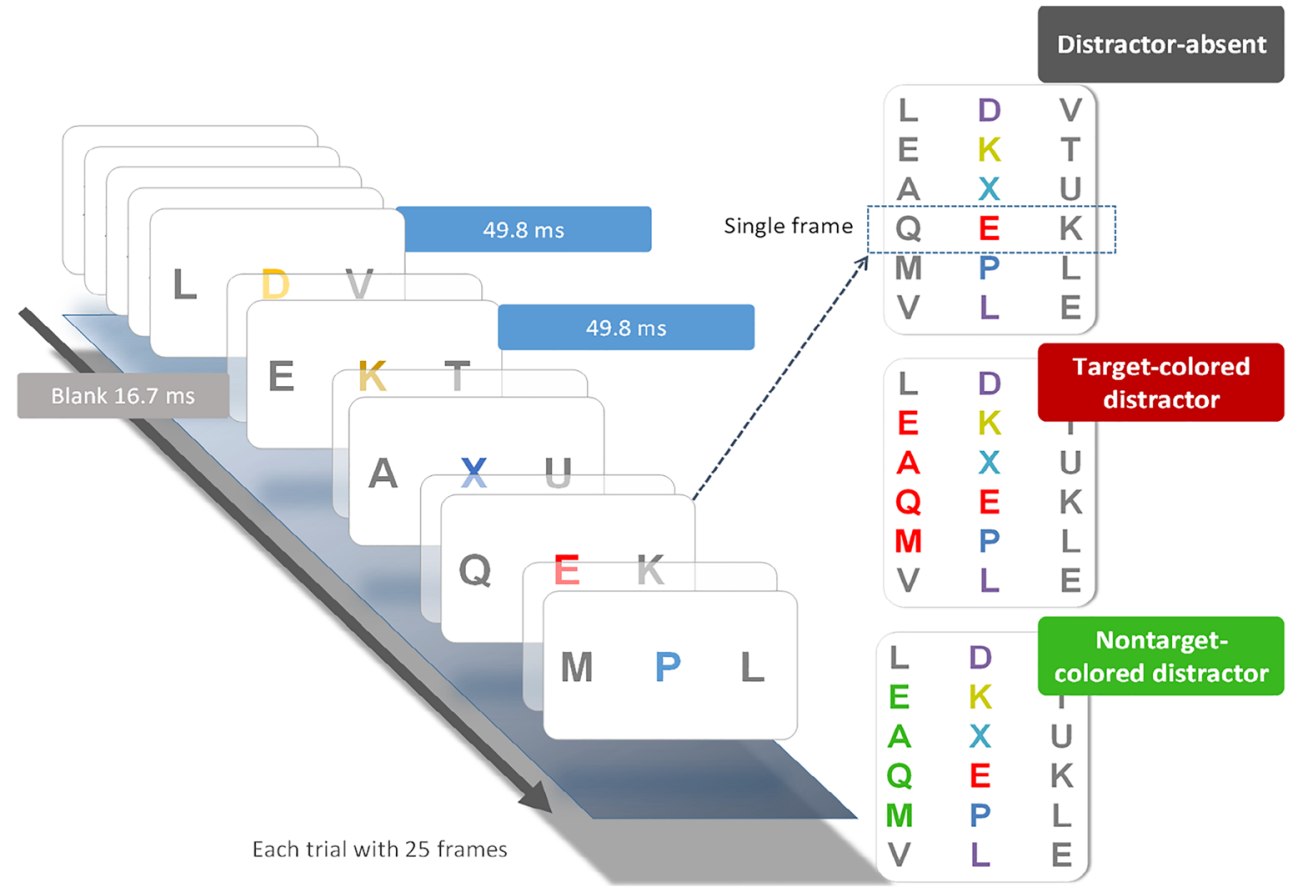

Fig. 1 The experimental conditions and procedure of the study are shown. Each letter stream contained 26 uppercase letters in a rapid serial order. Participants were required to search for the unique red letter in the middle stream. The peripheral flanker streams were mostly gray letters. In one-third of the trials, target-colored (i.e., red) distractors were presented in the flanker streams two frames before the onset of the central target letter and lasted until four frames after the offset of the target. In another one-third of the trials, these distractors were nontarget-colored (i.e., green). In the remaining one-third of the trials, the distractors are all gray (distractor-absent). (Color figure online)

\section{Procedure}

Before the experiment, participants received instruction regarding the study procedures and completed a consent form informing them of their right to withdraw from the experiment at any time.

For the task, participants were instructed to focus on the central target stream, while ignoring the peripheral distractor streams, and identify the red letter embedded in this stream. They were required to pressed the space key on the keypad to start each trial, which began with a centrally presented fixation cross for $500 \mathrm{~ms}$. Following the fixation cross, the rapid serial presentation of the three letter streams began. Each letter was presented for $50 \mathrm{~ms}$, followed by a $16.7 \mathrm{~ms}$ blank interval, such that a letter was presented every $66.7 \mathrm{~ms}$. They were required to press a button with their right index finger if the target letter was in the first eight of the English alphabet ( $A$ to $H$ ) and press another button with their right middle finger if the target letter was in the last eight of the English alphabet $(S$ to $Z$ ). The accuracy and response time data for all trials were recorded. After 20 practice trials, participants performed 240 trials over two sessions, with each session being 120 trials, with a rest break in between the sessions. The total duration of the task was between 20 and 25 minutes and it was presented in a dim, quiet room that was electrically shielded to enable EEG recording.

\section{EEG recording parameters}

For EEG recording (and task presentation) participants were seated in a comfortable armchair. Participants wore a 32-electrode arrangement digital EEG cap (Quik-Cap) with $\mathrm{Ag} / \mathrm{AgCl}$ sintered electrodes placed according to the international 10-20 system (FP1, FP2, F7, F3, Fz, F4, F8, FT7, FC3, FCz, FC4, FT8, T3, C3, Cz, C4, T4, TP7, CP3, CPZ, CP4, TP8, T5, P3, Pz, P4, T6, O1, Oz, $\mathrm{O} 2, \mathrm{~A} 1, \mathrm{~A} 2)$, off-line referenced to the left and right mastoid. The HEOL and HEOR channels positioned lateral to the left and right external canthi were used for detecting eye movements. The VEOU and VEOL channels positioned over and beneath the left eye were used for detecting eye movements. All scalp EEG electrode impedances were kept below $5 \mathrm{k} \Omega$. A Neuroscan amplifier (NuAmps) and Neuroscan 4.5 software were used for EEG acquisition with an analog $0.05-100 \mathrm{~Hz}$ bandpass filter and the signal was digitized at a $1000 \mathrm{~Hz}$ sampling rate.

EEG epochs from $100 \mathrm{~ms}$ before to $600 \mathrm{~ms}$ after the distractor onset were analyzed. Epochs with eye blink (EOG amplitude $> \pm 60 \mu \mathrm{V}$ in every channel) or with movements (EEG $> \pm 100 \mu \mathrm{V}$ ) were excluded from further analysis. Thirteen participants were excluded from further analyses because more than $30 \%$ of their total trials were rejected, leaving 35 participants (HTA: 16, LTA: 19). The 
epochs were averaged separately for each type of trial, according to the distractor type (absent, NTC, TC) and distractor location (right visual field, RVF; left visual field, LVF).

\section{Data Analysis}

\section{Behavioral data}

Forty-seven participants' data were included in the behavioral analysis. One participant's data were missing because of a malfunction of the experiment computer during the presentation of the task. Accuracy (ACC) and Mean reaction times (RTs) were used as dependent variables. For ACC analyses, all trials were analyzed. For RT analysis, only correct trials were included.

\section{EEG data}

The N2pc was quantified by subtracting the mean amplitudes of ipsilateral electrodes from contralateral electrodes with regards to the distractor location. Analysis of N2pc was confined to the lateral posterior electrodes, P3 and $\mathrm{P} 4$, where the N2pc component is maximal. The time window used for the N2pc was between 175-235 ms. This was selected based on first, an expected latency of around 200-300 ms for the N2pc maximum (Eimer, 1996; Luck \& Hillyard, 1994) and, following visual inspection of the data, computation of the voltage levels in a window from $155 \mathrm{~ms}$ to $255 \mathrm{~ms}$ (with a $60-\mathrm{ms}$ window and $10-\mathrm{ms}$ steps) to derive a window expected to contain the maximum component levels.

The statistical analysis of behavioral data and EEG data were broadly similar. To evaluate the effects of trait-anxiety level for the different distractor types, behavioral data and EEG data were subjected to a 2 (trait anxiety: HTA, LTA) $\times$ 3 (distractor type: absent, NTC, TC) mixed-design Analysis of Variance (ANOVA). All variables were within-subject, except for trait anxiety.

Some previous TMS and EEG/ERPs studies have reported a trend of lateralized effect of contingent attentional capture towards the left visual field and also dynamically interact with the trial history across two hemispheres (Chang et al., 2013; Chang et al., 2016). To examine the visual field asymmetry effect for the current experiment, a 2 (trait anxiety: HTA, LTA) $\times 2$ (distractor type: NTC, TC) $\times 2$ (distractor location: left visual field $(\mathrm{LVF})$, right visual field (RVF)) mixed-design ANOVA was conducted. The distractor-absent condition was excluded because there was no visual field difference for this condition. All variables were within-subject except for trait anxiety.

\section{Results}

\section{Accuracy}

Two-way mixed ANOVA revealed a significant main effect of distractor type, $F(2,90)=67.615, p<.001$, and post hoc paired-samples $t$-test comparisons revealed worse performance during the TC condition compared to the absent condition, $t(46)=9.216, p<.001$, and the NTC condition, $t(46)=$ 9.667, $p<.001$. There was no difference between the absent condition and NTC condition, $t(46)=0.11, p=.912$. The main effect of trait anxiety and the interaction with distractor type was not statistically significant, $F(2,90)=0.616, p=.543$ (see Fig. 2).

Three-way mixed ANOVA revealed a significant main effect of distractor location, $F(2,90)=11.593, p<.001$, and post hoc paired-samples $t$-test comparisons revealed that accuracy from left visual field was lower than right visual field, $t(46)=$ $3.442, p<.001$. The analysis also showed a significant main effect of distractor type, $F(1,45)=91.907, p<.001$. The main effects of trait anxiety and all other interactions were not statistically significant $(p>.05)$.

\section{Reaction times}

Two-way mixed ANOVA revealed a significant main effect of distractor type, $F(2,90)=31.735, p<.001$. Post hoc pairedsamples $t$-test comparisons showed slower performance during the TC condition compared to the absent condition, $t(46)=-5.502, p<.001$, and the NTC condition, $t(46)=-$ $6.384, p<.001$. There was no difference between the absent condition and the NTC condition, $t(46)=1.090, p=.282$. The main effects of trait anxiety, and all the other interactions were not statistically significant ( $p>.05$; see Fig. 3).

A three-way mixed ANOVA revealed a significant main effect of distractor location, $F(2,90)=6.987, p<.05$. Post hoc paired-samples $t$-test comparisons showed that reaction time for the left visual field was slower than for the right visual field distractors, $t(46)=2.577, p<.05$. The analysis also showed a significant main effect of distractor type, $F(1$, $45)=39.874, p<.001$. Post hoc paired-samples $t$-test comparisons showed slower performance during the TC condition compared to the NTC condition, $t(46)=-6.384, p<.001$. There was also a significant interaction between distractor location and trait anxiety, $F(1,45)=5.212, p<.05$. The follow-up two-way mixed-model ANOVA analysis revealed a significant main effect of visual field, $F(1,23)=30.570$, $p<.001$, for high trait anxiety, but not for low trait anxiety, $F(1,22)=0.039, p=.845$ (see Fig. 4). Further analysis showed that participants in the high-trait-anxiety group took more time when the distractor was located in left visual field than when it was in the right visual field for both the NTC, $t(23)=4.752, p<.001$, and the TC conditions, $t(23)=2.396$, 
HTA

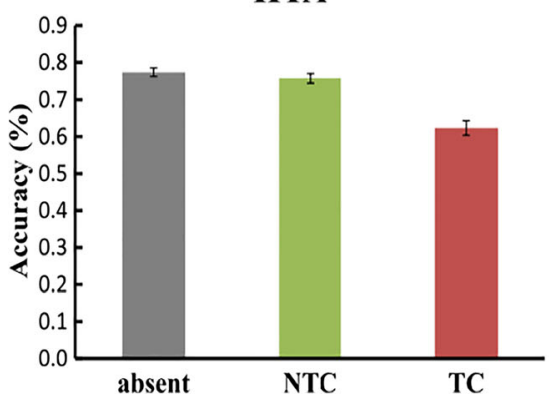

LTA

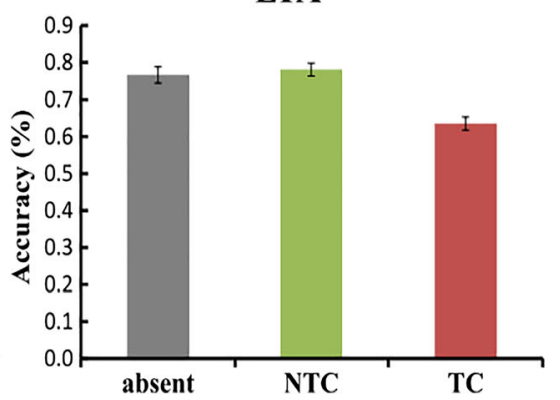

Fig. 2 Mean accuracy as a function of trait anxiety level and distractor type. Both high trait anxiety (HTA) and low trait anxiety (LTA) showed a significant contingent capture effect (lower accuracy of TC distractor condition). Error bars denote \pm 1 SEM. (Color figure online)

$p<.05$ (see Fig. 5). All the other interactions were not statistically significant $(p>.05)$.

\section{Electrophysiological results}

Figure 6 shows the ERPs obtained from electrodes P3/P4 contralateral to the three types of distractors (solid lines) and ipsilateral to the three types of distractors (dashed lines) during trials. The left panel shows the ERPs obtained from the highanxiety group and the right panel shows the ERPs obtained from the low-anxiety group. This figure shows that N2pc is larger in the TC condition than in the other two conditions and the $(\mathrm{N} 2 \mathrm{pc})$ values are larger in the high-anxiety group than in the low-anxiety group (see Fig. 6b). This can be seen more clearly in the illustrations of the difference waveforms in Fig. 7.

A two-way mixed-design ANOVA of difference waveforms revealed a significant main effect of distractor type, $F(2,66)=6.77, p<.05$, and also a significant two-way interaction between Trait Anxiety $\times$ Distractor Type, $F(2,66)=$ $3.399, p<.05$.

To explore the two-way interaction, separate repeatedmeasures ANOVAs were conducted for both of the trait anxiety groups, with distractor type as a within-participant factor. The high-anxiety group showed a significant main effect of distractor type, $(2,30)=10.539, p<.001$, whereas the low-

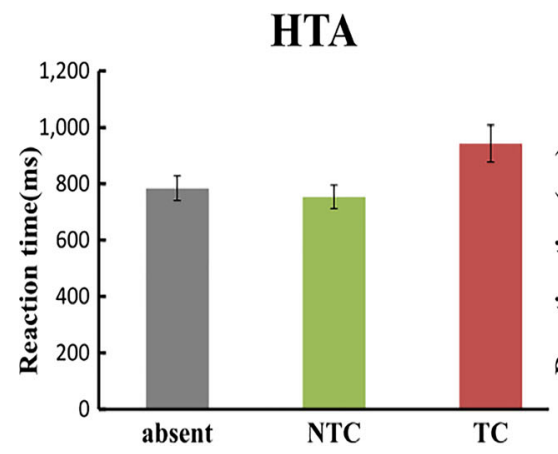

Fig. 3 Mean reaction time as a function of trait anxiety level and distractor type. Both high trait anxiety (HTA) and low trait anxiety (LTA) showed a significant contingent capture effect (lower accuracy anxiety group did not, $F(2,36)=0.97, p=.389$. Post hoc tests showed a significant difference between the TC condition and the absent condition, $t(15)=4.710, p<.05$. A significant difference was also found between the NTC condition and the absent condition, $t(15)=2.77, p=.014$. There was no significance difference between the NTC condition and TC condition, $t(15)=2.00, p=.064$. Furthermore, we found no significant differences between the low and high-anxiety groups for the absent conditions, $t(33)=1.976, p=.057$; TC, $t(33)=-$ $1.48, p=.15$; or NTC, $t(33)=-1.39, p=.17$ (see Fig. 7).

A three-way mixed-design ANOVA revealed a significant main effect of the distractor type, $F(1,33)=6.335, p<.05$. Post hoc paired-samples $t$-test comparisons showed that the N2pc was larger in the TC condition than in the NTC condition, $t(34)=2.506, p<.05$. The main effects of trait anxiety and distractor location and all other interactions were not statistically significant $(p>.05)$.

\section{Discussion}

The present study focused on investigating the effects of differences in trait anxiety on contingent attentional capture in a nonthreatening situation and also on the investigation of the effects of anxiety on involuntary attention asymmetry. Overall, the behavioral and electrophysiological results

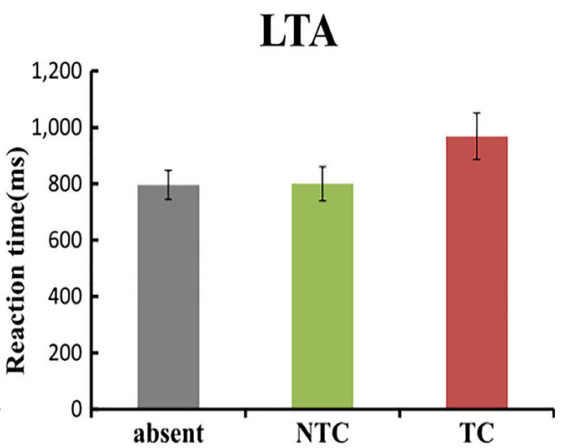

for the TC distractor condition). Error bars denote \pm 1 SEM. (Color figure online) 


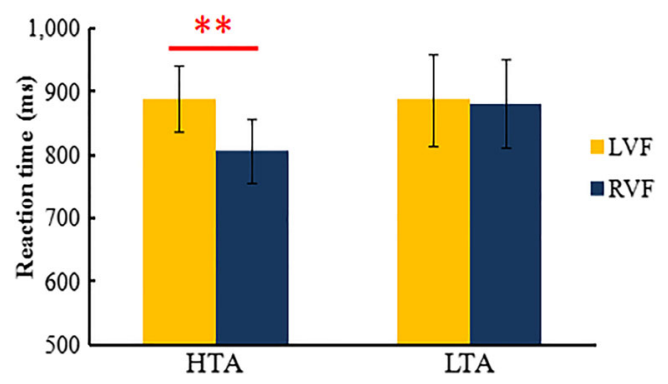

Fig. 4 Mean reaction time as a function of anxiety level and distractor location. A visual field asymmetry effect was found in the high trait anxiety group but not in the low-anxiety group. Error bars denote \pm 1 SEM. $* * p<.001$. (Color figure online)

showed a larger contingent capture effect in the target colored distractor condition (TC) compared to both the nontarget colored distractor (NTC) and distractor-absent conditions (Absent). These behavioral patterns replicated Chang et al.'s previous work in investigating contingent attentional capture in the general population (Chang et al., 2013; Chang et al., 2016). In addition, the electrophysiological results showed that the high trait anxiety group had a larger N2pc in the TC distractor and the NTC distractor conditions compared to the distractor-absent condition. However, no difference in N2pc was seen in the low-anxiety group across different conditions. Another group difference was found in the visual field asymmetry of the behavioral results. A longer reaction time was seen in high-anxiety individuals when distractors were presented in the left visual field than in the right visual field. Such an effect was not seen in low-anxiety individuals.

The results of a larger N2pc for the TC and NTC conditions relative to distractor-absent condition in high-anxiety individuals, in comparison to no differences in low-anxiety individuals, may indicate that high-anxiety individuals are limited in allocating the attention to the target efficiently, a possibility consistent with attentional control theory. However, we also saw automatic attentional capture was higher in the highanxiety group. Sawaki and Luck (2010) proposed a suppression mechanism is evoked to prevent attentional capture by salient but irrelevant distractors. The N2pc results in the present study may indicate that the high trait anxiety individuals could not preclude the allocation of the attention to the distractors irrespective of whether they were relevant or irrelevant. In other words, the high-trait-anxiety individuals could not suppress selecting the distractors, whereas the low-anxiety individuals could do so. Sawaki \&and Luck (2010) also addressed whether the suppression mechanism involves topdown control. The results here showed that anxiety might interfere with the top-down control and further affect the process of suppressing the distractors.

Behavioral results revealed a visual field asymmetry for high-trait-anxiety individuals. They showed a stronger capture effect when distractors were presented in the left visual field than when they were presented in the right visual field. Such an effect was not seen in low-anxiety individuals. This visual field asymmetry effect may reflect the different nature of processing between the two visual fields. Previous behavioral studies found that salient, target-relevant distractors presented in the left visual field resulted in a larger contingent attentional capture effect (Burnham, Rozell, Kasper, Bianco, \& Delliturri, 2011; Du \& Abrams, 2010). In addition, neurologically healthy participants tend to bisect lines to the left in line bisection tasks (Bowers \& Heilman, 1980; McCourt \& Garlinghouse, 2000), a phenomenon called pseudoneglect. These leftward asymmetry effects are widely observed across different tasks, which may reflect a right-hemisphere specialization (Corbetta \& Shulman, 2002). This fits with the model proposed of Corbetta and Shulman (2002), which claimed the ventral network is largely right lateralized. In addition, based on attentional control theory (Eysenck et al., 2007), another assumption is that anxiety increases the activation of the ventral network and decreases the activation of the dorsal frontal network resulting in reducing the inhibition of relevant distractors. Right hemisphere dominance of the ventral network and the increasing activation of the ventral network might lead to the leftward visual field asymmetry effect in the high trait anxiety group.
HTA

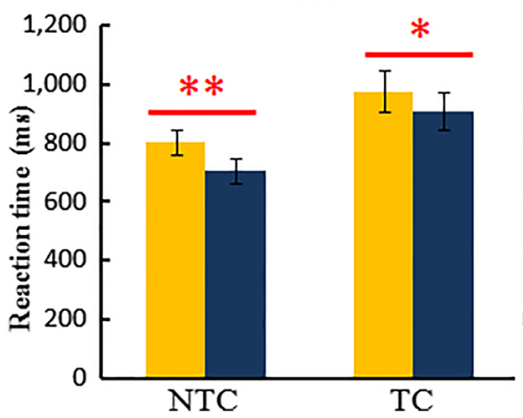

Fig. 5 Mean reaction time as a function of distractor location and distractor type, and whether trait anxiety was high (left panel) or low (right panel). High trait anxiety (HTA) individuals took more time to
LTA

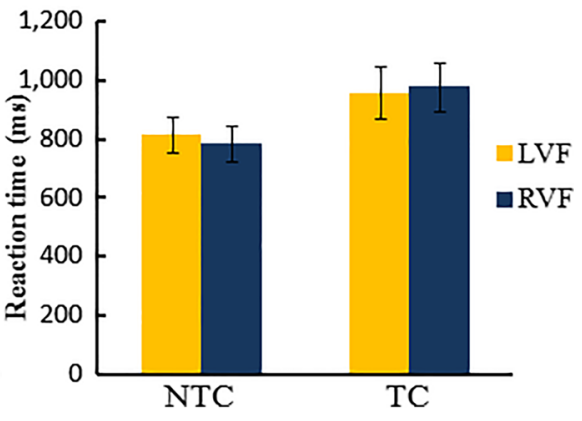

respond when the distractor was presented in the left visual field compared to the right visual field both in the TC and the NTC conditions. Error bars denote \pm 1 SEM. $* p<.05 . * * p<.001$. (Color figure online) 

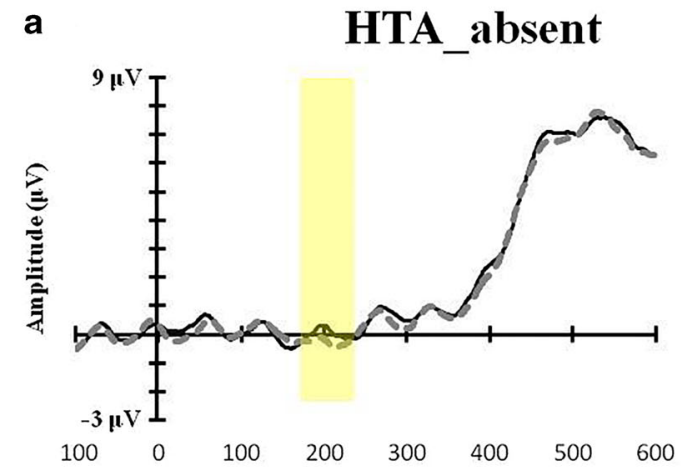

HTA_NTC

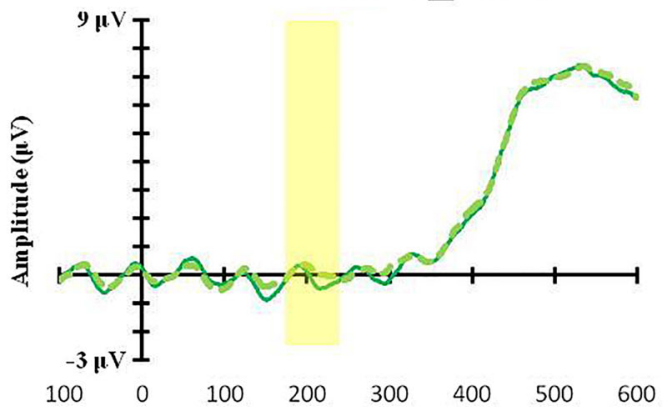

HTA_TC

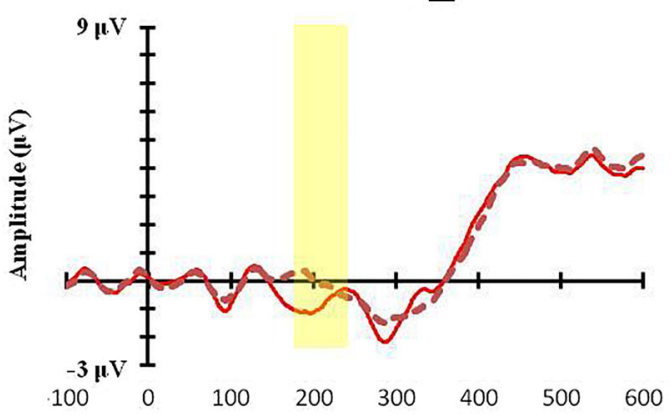

b

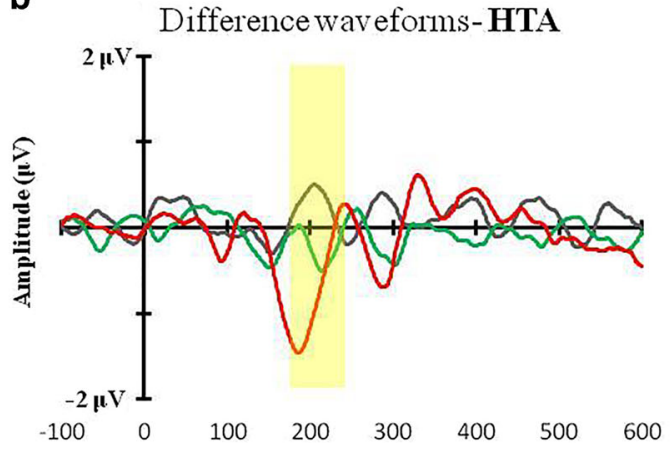

Fig. 6 Grand averaged ERPs and difference waveforms for the three distractor types for the high and low trait anxiety groups. a Grand averaged ERPs obtained at electrodes P3/P4 contralateral (solid line) and ipsilateral (dashed line) for the three types of distractors for the high-anxiety group (HTA, left panel) and for the low-anxiety group

In addition, several TMS studies have explored hemispheric asymmetry within each network. The top-down network is considered lateralized in the human brain, which is consistent

\section{LTA absent}

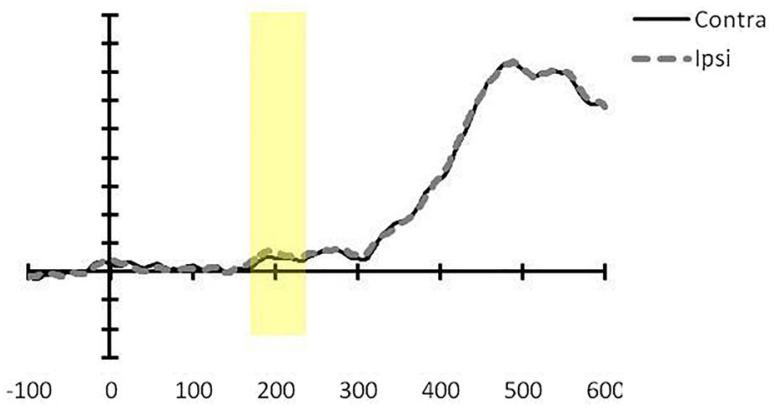

LTA_NTC

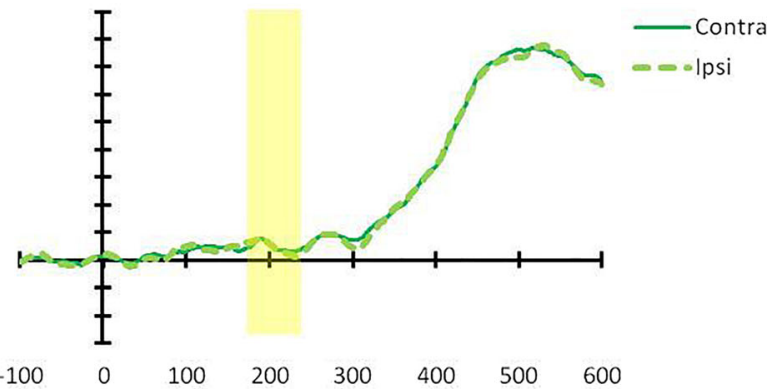

LTA_TC

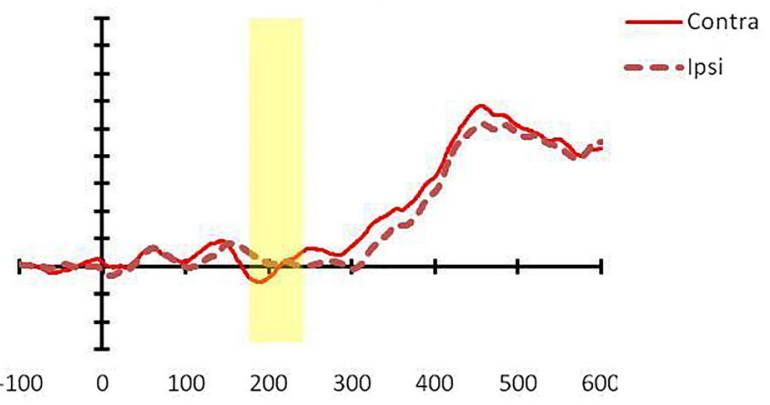

Difference wav eforms-LTA

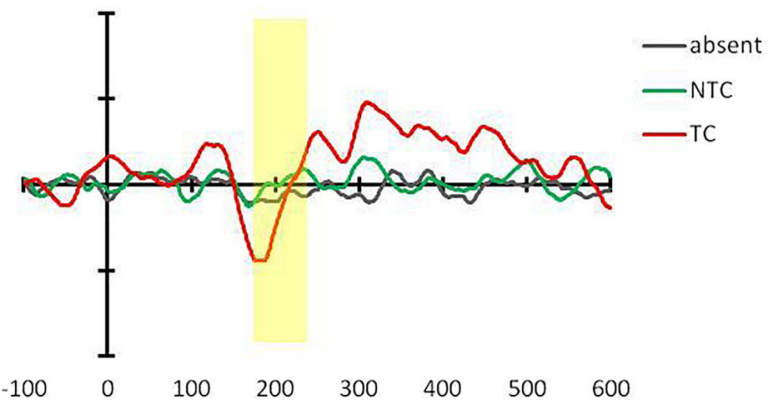

(LTA, right panel). b Difference waveforms obtained by subtracting ERPs at electrodes ipsilateral to the three types of distractors from those from contralateral electrodes for trials for the high-anxiety group (HTA, left panel) and for the low-anxiety group (LTA, right panel). (Color figure online)

with, for example, the evidence of dominance in the right frontal eye field (FEF), compared to the left, for visuospatial processing (Grosbras \& Paus, 2002; Silvanto, Lavie, \& Walsh, 

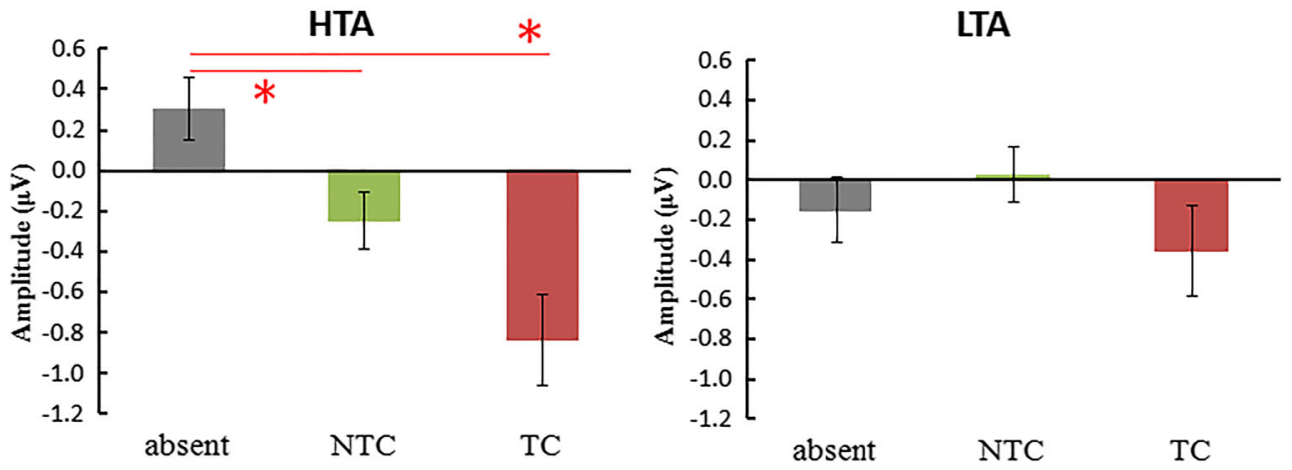

Fig. 7 N2pc values as a function of distractor type, and whether trait anxiety was high (left panel) or low (right panel). The contingent capture effect was seen in the HTA group, but not the LTA group. Error bars denote $\pm 1 S E M . * p<.05$. (Color figure online)

2006). Grosbras and Paus (2002) applied TMS on FEF during a visuospatial attention task, with an interference in the shift of attention occurring only when applying TMS stimulation over the right FEF. Likewise, the ventral network is thought to show a similar right dominance for this type of process. Chang et al. (2013) applied theta burst TMS over FEF and TPJ in order to investigate the effects of contingent attentional capture during the rapid serial visual presentation (RSVP) task. Right FEF TMS was found to enhance the performance in the task-irrelevant distractor condition, consistent with an increase of attention to the target. In contrast, right TPJ TMS influenced contingent attentional capture, with a significant decrease of performance for the task-relevant distractor condition, whereas left TPJ TMS did not produce such an effect. In a recent ERP/EEG study in which the identical RSVP paradigm was deployed (Chang et al., 2016), the sophisticated interaction between visual fields in contingent attentional capture has been revealed with the evidence that theta band oscillation started from the left hemisphere in the first section of the task then gradually propagating to the right hemisphere with the increment of trial exposure to the participant. This pattern of EEG results has demonstrated the dynamic features of interhemispheric intercommunication in the deployment of attention. It is clear that more studies are needed to elucidate the characteristics of attentional capture and reorienting.

In the present study, a discrepancy between the behavioral and ERP results was found. An interaction between distractor type (i.e., TC, NTC) and distractor absent and trait anxiety (i.e., high and low anxiety) was observed in the ERP results, whereas this type of interaction was not seen in the behavioral results. Such phenomena have been observed in a previous study. Dennis and Chen (2009) examined the Attention Network Test (ANT) in trait anxiety while the ERP was recorded. No differences were seen between high- and low-traitanxiety groups in reaction times, but there was a decreased $\mathrm{N} 2$, which reflects demands in processing conflict or inhibition, in high-anxiety participants. The interpretation proposed was that compensatory strategies (i.e., increased a strength of processing resources) might be used by high-anxiety individuals and lead to comparable behavioral performance for both high- and low-anxiety groups. While a difference ERP-related measure, the N2pc, is considered here, it is possible that a similar explanation applies (i.e., there are mechanistic differences in how the two groups perform the task, reflected in the electrophysiology but not causing any behavioral difference).

Another discrepancy between the behavioral and ERP results was in the effects of visual field asymmetry. Although we found no significant effects of visual field asymmetry in the physiological results, several reasons may account for the effects of visual field asymmetry that were seen. First, we found an asymmetry effect in the reaction times whereas no such effect was found for accuracy. Taking these two aspects of behavioral performance together, it is possible that accuracy was preserved at a cost of processing speed. Effects on asymmetry may then not be present in the physiological data because of the mechanism by which this occurs. A supplementary reason may be a limit in the data due to the number of trials. While eighty trials were included in each condition, this number is halved when analyzing the N2pc for each visual field. In addition, because of artifacts in the data, some trials had to be rejected. Although there might be a trend for an effect in TC high-anxiety individuals, this was not reliable and showed no statistically significant effect.

Previous studies adopted the affective stimuli to examine the effects of different trait anxiety levels on attentional capture (Derakshan, Ansari, Hansard, Shoker, \& Eysenck, 2009; Reeck, LaBar, \& Enger, 2012). The studies found affective stimuli, especially threat stimuli, resulting in a larger capture effect in a high-trait-anxiety population. An attentional bias towards threat phenomenon has also been demonstrated using threat stimuli in numerous studies (Bar-Haim et al, 2007; Cisler \& Koster, 2010). However, the current study used nonthreatening stimuli and also found high-trait anxiety participants showed a larger capture effect when distractors were presented in the left visual field, which is shown in the behavioral results. 
The findings for attentional capture processing and electrophysiology for the high- and low-trait-anxiety groups might imply a difference in mechanisms or brain connections for these two groups even in nonemotion arousing states. The present study employed a RSVP task to test contingent capture, which is one measure of attentional control and the pattern of the current electrophysiological results in the RSVP task are consistent with an earlier report by Chang et al. (2016). In order to gain a clearer indication of the importance of anxiety relative to a more independent measure of attentional control, future work might benefit from the use measures such as those provided by the Attentional Control Questionnaire or use of the attentional network task.

In conclusion, the present study provides further information about the characteristics of attentional control in traitanxiety, especially regarding how this relates to contingent attentional capture. The high-anxiety population was found to have an imbalance in suppressing attention toward the distractors, and have difficulty in disengaging from both contingent stimuli or salient stimuli located in the left visual field, even though they were emotionally neutral. This phenomenon may increase danger in everyday life in situations where over engagement by information fitting into such a category might be a disadvantage (one example where this could be expected to occur frequently is when driving a car). These findings illustrate the necessity for further investigation of differences in neural mechanisms in anxious populations. Better knowledge of the mechanisms underlying these differences may offer insight into methods that may aid in their improvement.

Acknowledgments This work was supported by grants from the Ministry of Science and Technology, Taiwan, to C.H.J. (103-2410-H008-023-MY3, 104-2745-B-008-002), N.G.M. (104-2420-H-008-001MY2; 102-2410-H-008-021-MY3) and Academia Sinica theme project (AS-105-TP-C02-2) to C.H.J.

\section{Compliance with ethical standards}

Author contributions H.J.L., Y.C.T., C.F.C., W.K.L., N.G.M., and C.H.J. designed the experiments. C.F.C. programmed the experimental stimuli. Y.C.T. and C.F.C. collected data. H.J..L, Y.C.T., C.F.C., W.K.L., and C.H.J. analyzed the data. H.J.L., N.G.M., Y.C.T., and C.H.J. wrote the manuscript.

\section{References}

Bacon, W. F., \& Egeth, H. E. (1994). Overriding stimulus-driven attentional capture. Perception \& Psychophysics, 55(5), 485-496.

Bar-Haim, Y., Lamy, D., Pergamin, L., Bakermans-Kranenburg, M. J., \& van IJzendoorn, M. H. (2007). Threat-related attentional bias in anxious and nonanxious individuals: A meta-analytic study. Psychological Bulletin, 133(1), 1-24.

Bishop, S. J. (2009). Trait anxiety and impoverished prefrontal control of attention. Nature Neuroscience, 12, 1.
Bowers, D., \& Heilman, K. M. (1980). Pseudoneglect: Effects of hemispace on a tactile line bisection task. Neuropsychologia, 18(4-5), 491-498.

Burnham, B. R., Rozell, C. A., Kasper, A., Bianco, N. E., \& Delliturri, A. (2011). The visual hemifield asymmetry in the spatial blink during singleton search and feature search. Brain and Cognition, 75(3), 261-272.

Chang, C. F., Hsu, T. Y., Tseng, P., Liang, W. K., Tzeng, O. J., Hung, D. L., \& Juan, C. H. (2013). Right temporoparietal junction and attentional reorienting. Human Brain Mapping, 34(4), 869-877.

Chang, C. F., Liang, W. K., Lai, C. L., Hung, D. L., \& Juan, C. H. (2016). Theta oscillation reveals the temporal involvement of different attentional networks in contingent reorienting. Frontiers in Human Neuroscience. doi:10.3389/fnhum.2016.00264

Chung, S. K., \& Long, C. F. (1984). A study of the revised State-Trait Anxiety Inventory. Psychological Testing, 31, 27-36.

Cisler, J. M., \& Koster, E. H. (2010). Mechanisms of attentional biases towards threat in the anxiety disorders: An integrative review. Clinical Psychology Review, 30(2), 203-216.

Corbetta, M., Patel, G., \& Shulman, G. L. (2008). The reorienting system of the human brain: From environment to theory of mind. Neuron, $58(3), 306-324$.

Corbetta, M., \& Shulman, G. L. (2002). Control of goal-directed and stimulus-driven attention in the brain. Nature Reviews Neuroscience, 3, 201-215.

Dennis, T. A., \& Chen, C. C. (2009). Trait anxiety and conflict monitoring following threat: An ERP study. Psychophysiology, 46(1), 122131.

Derakshan, N., Ansari, T. L., Hansard, M., L, S., \& Eysenck, M. W. (2009). Anxiety, inhibition, efficiency, and effectiveness: An investigation using antisaccade task. Journal of Experimental Psychology, 56(1), 48-55.

Derryberry, D., \& Reed, M. A. (2002). Anxiety-related attentional biases and their regulation by attentional control. Journal of Abnormal Psychology, 111(2), 225-236.

Du, F., \& Abrams, R. A. (2010). Visual field asymmetry in attentional capture. Brain and Cognition, 72(2), 310-316.

Eimer, M. (1996). The N2pc component as an indicator of attentional selectivity. Electroencephalography and Clinical Neurophysiology, 99(3), 225-234.

Eysenck, M. W., Derakshan, N., Santos, R., \& Calvo, M. G. (2007). Anxiety and cognitive performance: Attentional control theory. Emotion, 7(2), 336-353.

Folk, C. L., Remington, R. W., \& Johnston, J. C. (1992). Involuntary covert orienting is contingent on attentional control settings. Journal of Experimental Psychology, 18, 1030-1044.

Gibson, B. S., \& Kelsey, E. M. (1998). Stimulus-driven attentional capture is contingent on attentional set for displaywide visual features. Journal of Experimental Psychology: Human Perception and Performance, 24(3), 699-706.

Grosbras, M. H., \& Paus, T. (2002). Transcranial magnetic stimulation of the human frontal eye field: Effects on visual perception and attention. Journal of Cognitive Neuroscience, 14, 1109-1120.

James, W. (1890). Principles of psychology. New York: Holt.

Kim, M. J., \& Whalen, P. J. (2009). The structural integrity of an amygdala-prefrontal pathway predicts trait anxiety. The Journal of Neuroscience, 29(37), 11614-11618.

Luck, S. J., \& Hillyard, S. A. (1994). Spatial filtering during visual search: Evidence from human electrophysiology. Journal of Experimental Psychology: Human Perception and Performance, 20(5), 1000-1014.

MacLeod, C., \& Mathews, A. (1991). Biased cognitive operations in anxiety: Accessibility of information or assignment of processing priorities? Behaviour Research and Therapy, 29(6), 599-610.

McCourt, M. E., \& Garlinghouse, M. (2000). Asymmetries of visuospatial attention are modulated by viewing distance and visual field 
elevation: Pseudoneglect in peripersonal and extrapersonal space. Cortex, 36(5), 715-731.

Moser, J. S., Becker, M. W., \& Moran, T. P. (2012). Enhanced attentional capture in trait anxiety. Emotion, 12(2), 213-216.

Posner, M. I., \& Petersen, S. E. (1990). The attention system of the human brain. Annual Review of Neuroscience, 13, 25-42.

Qi, S., Ding, C., \& Li, H. (2014). Neural correlates of inefficient filtering of emotionally neutral distractors from working memory in trait anxiety. Cognitive, Affective, \& Behavioral Neuroscience, 14(1), 253-265.

Reeck, C., LaBar, K. S., \& Egner, T. (2012). Neural mechanisms mediating contingent capture of attention by affective stimuli. Journal of Cognitive Neuroscience, 24(5), 1113-1126.

Rissman, J. A., \& Zanto, T. P. (2015). Top-down suppression. Brain Mapping: An Encyclopedic Reference, 3, 261-267.

Ruz, M., \& Lupiáñez, J. (2002). A review of attentional capture: On its automaticity and sensitivity to endogenous control. Psicológica, 23, 283-309.

Sawaki, R., Geng, J. J., \& Luck, S. J. (2012). A common neural mechanism for preventing and terminating the allocation of attention. The Journal of Neuroscience, 32, 10725-10736.

Sawaki, R., \& Luck, S. J. (2010). Capture versus suppression of attention by salient singletons: Electrophysiological evidence for an automatic attend-to-me signal. Attention, Perception, \& Psychophysics, $72(6), 1455-1470$.
Serences, J. T., Shomstein, S., Leber, A. B., Golay, X., Egeth, H. E., \& Yantis, S. (2005). Coordination of voluntary and stimulus-driven attentional control in human cortex. Psychological Science, 16(2), 114-122.

Silvanto, J., Lavie, N., \& Walsh, V. (2006). Stimulation of the human frontal eye fields modulates sensitivity of extrastriate visual cortex. Journal of Neurophysiology, 96, 941-945.

Sylvester, C. M., Corbetta, M., Raichle, M. E., Rodebaugh, T., Schlaggar, B. L., Sheline, Y. I., \& Lenzel, E. J. (2012). Functional network dysfunction in anxiety and anxiety disorders. Trends in Neurosciences, 35(9), 527-535.

Vrticka, P., Sander, D., \& Vuilleumier, P. (2013). Lateralized interactive social content and valence processing within the human amygdala. Frontiers in Human Neuroscience, 6(358). doi:10.3389/fnhum. 2012.00358

Williams, J. M., Watts, F. N., MacLeod, C., \& Mathews, A. (1988). Cognitive psychology and emotional disorder. Chichester: Wiley.

Woodman, G. F., \& Luck, S. J. (1999). Electrophysiological measurement of rapid shifts of attention during visual search. Nature, 400(6747), 867-869.

Yantis, S., \& Jonides, J. (1990). Abrupt visual onsets and selective attention: Voluntary versus automatic allocation. Journal of Experimental Psychology: Human Perception and Performance, 16(1), 121-134. 